TITLE:

\title{
Eigenvalue sensitivity analysis capabilities with the differential operator method in the superhistory Monte Carlo method
}

\author{
AUTHOR(S): \\ Yamamoto, Toshihiro
}

\section{CITATION:}

Yamamoto, Toshihiro. Eigenvalue sensitivity analysis capabilities with the differential operator method in the superhistory Monte Carlo method. Annals of Nuclear Energy 2018, 112: $150-157$

\section{ISSUE DATE:}

2018-02

URL:

http://hdl.handle.net/2433/234645

\section{RIGHT:}

(c) 2018. This manuscript version is made available under the CC-BY-NC-ND 4 . O license

http://creativecommons.org/licenses/by-nc-nd/4.0/; The full-text file will be made open to the public on 01 Februan 2020 in accordance with publisher's 'Terms and Conditions for Self-Archiving'.; This is not the published version. Please cite only the published version.; この論文は出版社版でありません。引用の際には出版社版をご確認ご利用ください。 


\title{
Eigenvalue sensitivity analysis capabilities with the differential operator
} method in the superhistory Monte Carlo method

\section{Toshihiro Yamamoto*}

\begin{abstract}
Research Reactor Institute, Kyoto University, 2 Asashiro Nishi, Kumatori-cho,
\end{abstract} Sennan-gun, Osaka, 590-0494, Japan

\begin{abstract}
This paper applies the first-order differential operator method to the Monte Carlo

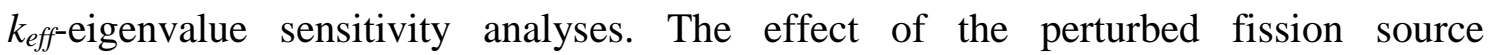
distribution due to the change of a cross section on the sensitivity coefficients can be accurately estimated by introducing the source perturbation iteration method. However, a prohibitively huge memory is required for the source perturbation iteration method if a large number of sensitivity coefficients are calculated at the same time. For a reduction of the memory requirements, the superhistory method is applied to incorporate the effect of the source perturbation into the differential operator method for sensitivity analyses. In the superhistory method, one source particle and its progenies are followed over super-generations within one cycle calculation. It is not necessary to wait or store a large amount of information until all histories in each cycle are terminated. Although the superhistory method increases the variance of the sensitivity coefficients with the super-generation, the memory requirement can be dramatically reduced by introducing the superhistory method. The first-order differential operator method combined with the superhistory method is verified through some numerical examples where a localized cross section change significantly affects the sensitivity coefficients.
\end{abstract}

* Corresponding author. Tel:+81 72451 2414; fax:+81 724512658

E-mail address: toshihiro.yamamoto223@gmail.com (T. Yamamoto) 
2 Keywords: Monte Carlo; Sensitivity coefficient; Differential operator; Superhistory 3

\section{Introduction}

There has been a growing interest in sensitivity and uncertainty analysis of

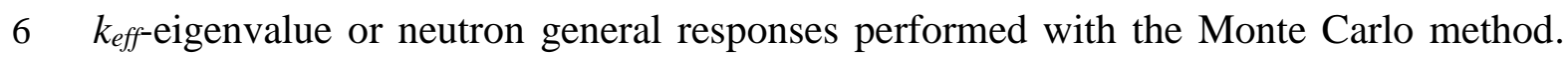

7 Additionally, there has been much research and techniques developed to date. The

8 sensitivity analysis methods are now implemented into production-level Monte Carlo

9 codes such as SCALE (Rearden, 2004; Perfetti, 2012; Perfetti and Rearden, 2016),

10 MCNP (Kiedrowski et al., 2011; Kiedrowski and Brown, 2013), SERPENT (Aufiero et

11 al., 2015), MORET (Jinaphanh et al., 2016), McCARD (Shim and Kim, 2011), and

12 RMC (Qiu et al., 2015; Qiu et al., 2016a; Qiu et al., 2016b). The calculation of the

13 adjoint flux, which is necessary for sensitivity analysis, was considered difficult for the

14 continuous-energy Monte Carlo. The iterated fission probability (IFP) method was developed for estimating the adjoint flux in the continuous energy Monte Carlo and the method is now implemented in many Monte Carlo codes (Truchet, et al., 2015; Terranova and Zoia, 2017). The IFP method calculates the expected number of neutrons caused by a neutron at a location in phase space as the adjoint function. The contribution method, which was originally developed for shielding applications and is implemented in the SCALE code, determines the importance of an event by simulating secondary particles at the site of the event and tracking the number of fission neutrons created by each secondary particle (Williams, 1977). A method implemented in the SERPENT code is based on the "collision-history based method" where all cross sections involved in the sensitivity calculations are artificially increased. Another method that this paper focuses on is the differential operator method (Rief, 1984; McKinney and Iverson, 1996; Densmore et al., 1997; Nagaya and Mori, 2005; Raskach, 
1 2009; Raskach, 2010; Jinaphanh et al., 2016). The unique feature of the differential

2 operator method is that the calculation of the adjoint flux can be circumvented and the

3 first derivative of $k_{\text {eff }}$-eigenvalue with respect to nuclear data can be estimated directly.

4 Furthermore, the differential operator method is applicable to estimating responses of

5 wide range of calculation characteristics: $k_{\text {eff }}$, reaction rates and their ratios both in the

6 eigenvalue problem and in the problem of a subcritical system driven by an external

7 neutron source (Raskach, 2010). On the other hand, the IFP method is applicable to

8 computing $k_{\text {eff }}$ derivatives and sensitivities only. The differential operator method has

9 been previously implemented in the MCNP code. However, the differential operator

10 method was replaced by another method; presumably, because it produces inaccurate

11 sensitivity coefficient estimates for complex systems. The sensitivity coefficient is the

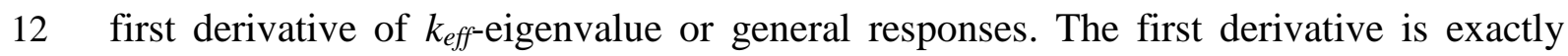

13 sampled in the differential operator method. Nevertheless, the inaccuracy in the

14 differential operator method is caused by neglecting the perturbation of the fission

15 source distribution that cannot be taken into account unless a special technique is employed for considering the perturbation. A method for implementing the source perturbation effect was developed in (Nagaya and Mori, 2005; Nagaya and Mori, 2011; Nagaya et al., 2015; Raskach, 2009). However, the method requires the iteration procedure similar to IFP to obtain the converged perturbed fission source distribution. The iteration procedure is similarly required in the IFP method where the fission chain is tracked for a number of generations to compute the adjoint-weighted tallies. The iteration procedure in the differential operator method or in the IFP method results in an increase in the memory requirements if the sensitivity coefficients of many isotopes, reactions, and fine energy groups are calculated at the same time.

Several techniques for reducing the huge memory requirements have been 
1 developed and installed in the Monte Carlo codes. In MCNP, a sparse data handling

2 scheme is employed, reducing the memory requirement by a factor of 10 to 100 for

3 many problems (Kiedrowski and Brown, 2013). In McCARD (Shim and Kim, 2011;

4 Choi and Shim, 2016a; Choi and Shim, 2016b), a memory-efficient adjoint estimation

5 method was developed by applying the IFP concept for the Monte Carlo Wielandt

6 method (Yamamoto and Miyoshi, 2004). In RMC (Wang, et al., 2015; Qiu et al., 2015;

7 Qiu et al., 2016a; Qiu et al., 2016b), the superhistory method (Brissenden and

8 Garlick,1986) as well as the Wielandt method was adopted to reduce the memory

9 consumption.

10 While the sensitivity analysis methods for generalized responses in the Monte

11 Carlo method have been developed (Choi and Shim, 2016b; Qiu, et al., 2016a; Perfetti 12 and Rearden, 2016; Aufiero et al., 2016), the present paper focuses on the sensitivity 13 analysis of $k_{\text {eff }}$ eigenvalue. This paper scrutinizes the source perturbation effect on a 14 sensitivity coefficient due to the change of nuclear data through the multi-group Monte

15 Carlo calculations. A method to include the source perturbation effect in the sensitivity coefficients and a memory reduction technique using the superhistory method are discussed in the following sections.

2. Methodology of $k_{\text {eff-eigenvalue sensitivity calculation with the differential }}$ operator method

\subsection{The differential operator method without perturbed source effect}

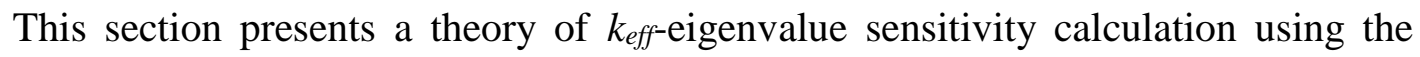
Monte Carlo differential operator method. The differential operator method for the perturbation calculation with the source perturbation being implemented was already established in previous research (Rief, 1984; Nagaya and Mori, 2005; Nagaya and Mori, 
1 method was expanded to the second and higher orders (Nagaya and Mori, 2011). In a

2 Monte Carlo code, MVP (Nagaya et al., 2015), the order of the differential operator

3 method was uniquely expanded to the 8 th order. The reactivity change due to a local

4 perturbation can be accurately obtained by the differential operator method by

5 introducing the source perturbation and by expanding the higher order Taylor series. For

6 sensitivity analyses, only the first-order derivatives are required. In this section, the

7 method to calculate the sensitivity coefficient is repeatedly presented as follows;

8 although, it is the duplication of the previously published papers. The formalism to

9 calculate the first derivative of $k_{\text {eff-eigenvalue with respect to a parameter was presented }}$

10 in detail in previous publications (e.g., Nagaya and Mori, 2005). This paper only

11 presents the minimum explanations for coding a Monte Carlo program to calculate the

12 first derivative of $k_{\text {eff-eigenvalue. }}$

13 The differential operator method scores an estimate of each differential coefficient

14 at each flight path or each collision point within a perturbed region. The estimates that are scored during the course of the random walk process are shown as follows. First, a particle starts from a fission source site $\mathbf{r}$. The angle is determined isotropically using a random number. The particle moves to a collision point $\mathbf{r}^{\prime}$ that is determined by the transport kernel:

$$
T\left(\mathbf{r} \rightarrow \mathbf{r}^{\prime}\right)=\Sigma_{t} \exp \left(-\Sigma_{t} s\right)
$$

where $\Sigma_{t}=$ the macroscopic total cross section, $s=$ the flight distance. When the particle travels a distance $s$ through the perturbed region and undergoes a collision, the weighting coefficient to be scored is

$$
\frac{1}{T} \frac{\partial}{\partial a} T\left(\mathbf{r} \rightarrow \mathbf{r}^{\prime}\right)=\frac{1}{\Sigma_{t}} \frac{\partial \Sigma_{t}}{\partial a}-s \frac{\partial \Sigma_{t}}{\partial a}
$$

where $a$ is a perturbation parameter. For simplicity, the variables for the energy and the direction are omitted. If the sensitivity coefficient with regard to a microscopic capture 
1 cross section of a nuclide $i$ is sought, $a=\sigma_{c, i}$ and $\partial \Sigma_{t} / \partial a=N_{i}$ where $\sigma_{c, i}=$ the

2 microscopic capture cross section of the nuclide $i, N_{i}=$ the atom number density of the

3 nuclide $i$. If $a=$ the macroscopic capture cross section $\Sigma_{c}, \partial \Sigma_{t} / \partial a=1$. If the particle

4 passes through the perturbed region without undergoing a collision, only the second

5 term on the right-hand side of Eq. (2), $-s \cdot \partial \Sigma_{t} / \partial a$, is scored.

6 Unless the particle is killed at the collision point, the particle undergoes a scattering

7 reaction. The weighting coefficient for the scattering kernel $\Sigma_{s} / \Sigma_{t}$ is

$$
\frac{\Sigma_{t}}{\Sigma_{s}} \frac{\partial}{\partial a} \frac{\Sigma_{s}}{\Sigma_{t}}=\frac{1}{\Sigma_{s}} \frac{\partial}{\partial a} \Sigma_{s}-\frac{1}{\Sigma_{t}} \frac{\partial}{\partial a} \Sigma_{t}
$$

9 where $\Sigma_{s}=$ the macroscopic scattering cross section.

The $k_{e f f}$-eigenvalue is the sum of $v \Sigma_{f} \cdot w / \Sigma_{t}$ at each collision point in a cycle

where $w=$ the weight of the colliding particle, $v=$ the number of neutrons per fission, and $\Sigma_{f}=$ the macroscopic fission cross section. Thus, the perturbation of $v \Sigma_{f}$ or $\Sigma_{t}$ contributes to the change of $k_{\text {eff. }}$ To include this effect in the sensitivity coefficient, the following weighting coefficient is scored at each collision:

$$
\frac{\Sigma_{t}}{v \Sigma_{f}} \frac{\partial}{\partial a} \frac{v \Sigma_{f}}{\Sigma_{t}}=\frac{1}{v \Sigma_{f}} \frac{\partial}{\partial a} v \Sigma_{f}-\frac{1}{\Sigma_{t}} \frac{\partial}{\partial a} \Sigma_{t} .
$$

The scorings of Eqs. (2), (3), and (4) are repeated at each flight and collision until the particle is discarded. As a result, the first derivative of the $k_{\text {eff-eigenvalue with }}$ respect to the perturbation parameter $a$ for the $m$ th particle history is given by

$$
\frac{\partial}{\partial a} k_{e f f, N P, m}=\sum_{i} \frac{v \Sigma_{f, i}}{\Sigma_{t, i}} w_{i} w_{N P, i}
$$

where $w_{i}=$ the particle weight of the $i$ th collision, and

$$
w_{N P, i}=\frac{1}{v \Sigma_{f, i}} \frac{\partial}{\partial a} v \Sigma_{f, i}-\frac{\delta_{a \Sigma_{s}}}{\Sigma_{t, i}}+\sum_{l=1}^{i} \frac{1}{\Sigma_{s, l}} \frac{\partial}{\partial a} \Sigma_{s, l}-\sum_{k} s_{k} \frac{\partial}{\partial a} \Sigma_{t, k},
$$

where $\delta_{a \Sigma_{s}}\left\{=0\right.$ if $a \neq \Sigma_{s},=1$ if $\left.a=\Sigma_{s}\right\}$. The subscript $N P$ denotes that Eq. (5) 
1 sections. The summation symbol on the right-hand side of Eq. (5) means that the

2 summation is carried out at every collision point during the $m$ th history. The second

3 term on the right-hand side of Eq. (6) means the sum of $1 / \Sigma_{s, l} \cdot \partial \Sigma_{s, l} / \partial a$ until the $i$ th

4 collision where $\Sigma_{s, l}$ is the macroscopic scattering cross section for the $l$ th scattering.

5 The last term on the right-hand side of Eq. (6) means the sum of $s_{k} \cdot \partial \Sigma_{t, k} / \partial a$ in the

$6 k$ th flight distance of the perturbed region until the $i$ th collision. The term

$7 \quad 1 / \Sigma_{t} \cdot \partial \Sigma_{t} / \partial a$ in Eq. (2) cancels out the same term in Eq. (3) or (4). Thus, the term

8 does not explicitly appear in Eq. (6). Eq. (6) is represented for some perturbation

9 parameters as follows:

$$
w_{N P, i}=\left\{\begin{array}{c}
-\sum_{k} s_{k}, \quad a=\Sigma_{c}, \\
-\frac{1}{\sum_{t, i}}+\sum_{l=1}^{i} \frac{1}{\Sigma_{s, l}}-\sum_{k} s_{k}, \quad a=\Sigma_{s}, \\
\frac{1}{\Sigma_{f, i}}-\sum_{k} s_{k}, \quad a=\Sigma_{f}, \\
1, \quad a=\chi_{g} .
\end{array}\right.
$$

11 Eq. (10) represents the sensitivity coefficient with respect to the fission spectrum in the gth group, and applies only when a particle starting from the fission site in the perturbed region belongs to the gth group. After all the particles starting from the fission source sites for one cycle are exhausted, the sensitivity coefficient in the cycle is calculated:

$$
\frac{\partial k_{e f f, N P}}{\partial a}=\frac{1}{M} \sum_{m=1}^{M} \frac{\partial}{\partial a} k_{e f f, N P, m},
$$

where $M=$ the number of particle histories in one cycle.

\subsection{The differential operator method with perturbed source effect}

A perturbation of a cross section changes the fission source distribution whose effect is not taken into account in Eq. (11). The fission source distribution $S\left(\mathbf{r}_{0}\right)$ is perturbed by the change of a cross section. The source perturbation effect of the first 
1 derivative of the $k_{e f f}$-eigenvalue in the $m$ th history in the $j$ th cycle is scored at each

2 collision point:

$$
\frac{\partial}{\partial a} k_{e f f, P S, m, j}=\sum_{i} \frac{v \Sigma_{f, i}}{\Sigma_{t, i}} w_{i} w_{P S, m, j, N},
$$

4 where $w_{P S, m, j, N}=$ the score for $1 / S\left(\mathbf{r}_{0}\right) \cdot \partial S\left(\mathbf{r}_{0}\right) / \partial a$ and it is obtained in the previous

5 cycle. $w_{P S, m, j, N}$ in Eq. (12) depends on the perturbed fission source distribution that

6 is caused by the change of the cross section. It needs to be calculated by an iteration

7 procedure. The subscript $N$ in $w_{P S, m, j, N}$ stands for an index of the iteration for the

8 source perturbation. The number of iteration $N$ should be as large as 10 for estimating

9 an accurate source perturbation effect (Nagaya and Mori, 2005). In the $j$ th cycle,

$10 w_{P S, m, j+1, n+1}$, which is used for the next $(j+1)$ th cycle, is calculated as follows. At a point where the $l$ th fission neutron in the $j$ th cycle is born in the $m$ th history, the following quantity is scored:

$$
\begin{gathered}
w_{l, j, n}^{f}=w_{N P, i, j}, \quad \text { for } n=1, \\
w_{l, j, n}^{f}=w_{N P, i, j}+w_{P S, m, j, n}, \text { for } \quad N \geq n \geq 2,
\end{gathered}
$$

where $w_{N P, i, j}$ is the same one as defined in Eq. (6) except that the cycle index $j$ is added. $w_{P S, m, j, n}$ is inherited from the previous $(j-1)$ th cycle. The subscript $n$ stands for the index of iteration for the source perturbation. In each cycle, $w_{l, j, n}^{f}$ is stored for $N \geq n \geq 1$ and $L \geq l \geq 1$ where $L=$ the total number of fission neutrons born in the $j$ th cycle. The memory requirement is $N \times L \times$ bytes per variable for each cross section and each energy group.

The number of fission neutrons for use as the source points in the next cycle is determined at the $i$ th collision point as:

$$
l=\frac{1}{k_{j-1}} \frac{v \Sigma_{f, i}}{\Sigma_{t, i}} w_{i}+\xi,
$$

24 where $k_{j-1}=k_{\text {eff }}$ calculated in the previous cycle, $\xi=$ pseudo random number between 0 and 1. At the end of the $j$ th cycle, $w^{f} l, j, n$ calculated by Eq. (13) or (14) is 
1 normalized and we obtain $w_{P S, l, j+1, n+1}$ for the next cycle:

$$
w_{P S, l, j+1, n+1}=w^{f} l, j . n-\frac{1}{L} \sum_{l}^{L} w^{f} l, j . n \text { for } L \geq l \geq 1 \text { and } N-1 \geq n \geq 1 .
$$

3 This normalization process is to keep the size of the sampling constant in each cycle. At

4 the end of the $j$ th cycle, the first derivative of the $k_{\text {eff-eigenvalue with respect to } a \text { with }}$

5 the source perturbation effect included is calculated:

$$
\frac{\partial k_{e f f, P S, j}}{\partial a}=\frac{1}{M} \sum_{m=1}^{M} \frac{\partial}{\partial a} k_{e f f, P S, m, j} .
$$

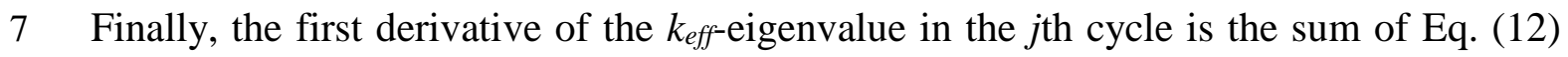

8 (without the source perturbation effect) and Eq. (17) (with the source perturbation 9 effect):

\subsection{Memory reduction with the superhistory method}

If the effect of the source perturbation is negligibly small, the differential operator method can omit the iteration procedure for the source perturbation and the memory requirement does not cause a significant problem. However, the effect of the source perturbation needs to be considered by introducing the iteration procedure like the IFP method. If the reactivity change due to the perturbation of cross sections is sought to be known, the memory requirement is (the number of iterations for source perturbation, $\sim 10) \times($ the number of histories per cycle $) \times($ the number of perturbed cross sections $) \times$ (bytes per variable). The memory requirement for a perturbation calculation is not serious, because the number of cross sections to be perturbed is not generally so large for the reactivity change. On the other hand, for a calculation of sensitivity coefficients, the number of cross sections is (the number of isotopes) $\times$ (the number of reactions) $\times$ (the number of energy groups). Thus, the memory requirement becomes prohibitively huge if the calculation is performed at one time. This is because $w^{f} l, j, n$ (defined in 
1 Eq. (13) or (14)) needs to be stored for $N \geq n \geq 1$ and $L \geq l \geq 1$ until $w_{P S, l, j+1, n+1}$

2 (defined in Eq. (16)) is obtained at the end of the cycle. In this section, the superhistory

3 method, which is adopted for memory reduction in a calculation of sensitivity

4 coefficients (Qiu et al., 2016a; Qiu, et al., 2016b), is introduced to exclude the memory

5 consuming iteration procedure. The superhistory method was originally invented to

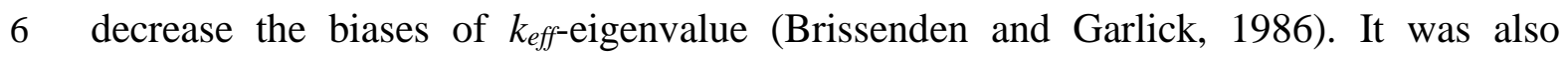

7 applied to accelerate the source convergence in $k_{\text {eff }}$ eigenvalue calculations (Blomquist

8 and Gelbard, 2002; She, et al., 2012). In the superhistory method, the fission neutrons in

9 a cycle are tracked over $N_{S}(>1)$ fission generations, which is called a "supergeneration".

10 The $k_{\text {eff }}$ calculated in the $N_{S}$ th supergeneration (i.e., the last supergeneration of the cycle)

11 is adopted as the $k_{\text {eff }}$ of the cycle, and the fission neutrons generated in the $N_{S}$ th

12 supergeneration are inherited to the next cycle as the fission sources.

13 The iteration procedure for the source perturbation shown in Sec. 2.2 can be

14 implemented into the information transfer process between supergenerations in the

15 superhistory method. At the first supergeneration of the $j$ th cycle, when the $l$ th fission

16 neutron is born at the $i$ th collision, $w_{N P, i, 1}$, which is defined in Eq. (6) for the $i$ th

17 collision in the history, is assigned to the fission neutron as

$$
w^{s} l, j, 1=w_{N P, i, 1},
$$

where $w_{l, j, 1}^{s}=$ the weighting coefficient of the $l$ th fission neutron in the first supergeneration of the $j$ th cycle. The number of fission neutrons in each supergeneration is determined using Eq. (15) as in the conventional power iteration method. The total number of fission neutrons in each supergeneration is nearly constant, because $v$ is divided by $k_{e f f}$ in the previous cycle in Eq. (15).

In the second supergeneration, $w^{s} l, j, 1$, which is assigned to the $l$ th source neutron, is further transferred to an $l^{\prime}$ th fission neutron for use in the third supergeneration as 


$$
w^{s} l^{\prime}, j, 2=w^{s} l, j, 1 .
$$

2 In this way, $w_{N P, i, 1}$ is transferred to a fission neutron in the next supergeneration. This

3 procedure is repeated until the final supergeneration of the $j$ th cycle. At the end of the

4 final supergeneration, the first derivative of $k_{\text {eff }}$ for the $m$ th superhistory in the $j$ th cycle

5 is given by

$$
\frac{\partial}{\partial a} k_{e f f, m, j}=\sum_{i} \frac{v \Sigma_{f, i}}{\Sigma_{t, i}} w_{i} w_{m, j, N}^{s},
$$

where the summation is performed at each collision point in the $N$ th supergeneration

8 and $w^{s}{ }_{m, j, N}$ is for the $m$ th superhistory in the $N$ th supergeneration. The first derivative

9 given by Eq. (20) implicitly consists of the sum of the following two terms defined by

10 Eqs. (5) and (12):

$$
\frac{\partial}{\partial a} k_{e f f, N P, m, j}+\frac{\partial}{\partial a} k_{e f f, P S, m, j}
$$

The final result of the first derivative of $k_{\text {eff }}$ that includes the source perturbation effect can be obtained one by one in each superhistory. Thus, it is not necessary to wait or store a large amount of information until all histories in each cycle are terminated. The memory requirement for the superhistory method can be reduced by a factor of (the number of histories per cycle) $\times$ (the number of iterations for source perturbation) compared to the method in Sec. 2.2. The normalization at the end of each supergeneration is performed by dividing the number of fission neutrons by $k_{\text {eff }}$ as seen in Eq. (15).

\section{Numerical tests for sensitivity coefficient calculations}

\subsection{Perturbation source method}

In this paper, some numerical examples for the calculations of $k_{\text {eff-sensitivity }}$ coefficients are presented using three-energy group calculations. Fig. 1 shows the geometry for the calculations is a two-dimensional rectangular shape. The inner and 
1 outer regions consist of a homogenized light-water moderated mixed oxide fuel rod 2 array and a homogenized $\mathrm{UO}_{2}$ fuel rod array, respectively. Table 1 shows the

3 three-energy group constants. The group constants are prepared with a standard thermal

4 reactor analysis code SRAC (Okumura et al., 2007). The sensitivity coefficients are

5 calculated with respect to the macroscopic cross sections or fission spectrum in the

6 inner region. The source perturbation effect on the sensitivity coefficients is emphasized,

7 because the perturbed cross section is localized within the inner region, which may be a

8 good example for testing the capability of the source perturbation.

The reference calculations for the sensitivity coefficients are performed with a 11 discrete ordinates transport code DANTSYS (Alcouffe et al., 1995) using the same 12 group constants. The three-energy group forward and adjoint fluxes are calculated with 13 the $S_{n}$ order 8 . Then, the sensitivity coefficients are calculated based on the linear 14 perturbation theory:

$$
S_{x}=\frac{x}{k_{e f f}} \frac{d k_{e f f}}{d x}=\frac{\left\langle\boldsymbol{\Phi}^{*} x \frac{d F}{d x} \boldsymbol{\Phi}\right\rangle+k_{e f f}\left\langle\boldsymbol{\Phi}^{*} x \frac{d S}{d x} \boldsymbol{\Phi}\right\rangle-k_{\text {eff }}\left\langle\boldsymbol{\Phi}^{*} x \frac{d \Sigma_{t}}{d x} \boldsymbol{\Phi}\right\rangle}{\left\langle\boldsymbol{\Phi}^{*} F \boldsymbol{\Phi}\right\rangle},
$$

where $x=$ a cross section, $\boldsymbol{\Phi}=$ the forward flux, $\boldsymbol{\Phi}^{*}=$ the adjoint flux, \langle\rangle$=$ the

17 integration over all phase space, $F=$ the production operator, and $S=$ the scattering operator.

19 The sensitivity coefficients to the capture cross section, to the fission cross section,

20 to the scattering cross section, and to the fission spectrum are calculated with DANTSYS and the Monte Carlo method. They are compared in Tables 2, 3, 4, and 5, respectively. In these tables, the row of "MC/DANTSYS" shows the ratio of the sensitivity coefficient of the Monte Carlo method to that of DANTSYS. The sensitivity coefficients to the fission spectrum are unconstrained ones. The Monte Carlo 
1 calculations are performed with an in-house research-purpose program developed by the

2 author, which is only available for the purpose of this study. The $k_{\text {eff }}$ is calculated with

3 the track length estimator. The number of histories per cycle is 60,000 and the total

4 active cycles after skipping the initial 20 cycles are 2,000. The program employs the

5 implicit capture with Russian roulette. When a particle's weight falls below 0.1, Russian

6 roulette game is played. The number of iterations for the source perturbation is 14 .

7 Tables 2, 3, 4, and 5 show the results of the Monte Carlo method, which are the

8 coefficients without the source perturbation and the coefficients with the source

9 perturbation. Table 4 shows the effect of the source perturbation is notable especially in

10 the sensitivity coefficient to the scattering cross section. The sensitivity coefficient with

11 the source perturbation is larger than that without the source perturbation. Fig. 2 shows

12 the sensitivity coefficient with the source perturbation, $S_{\mathrm{ps}}$ to the scattering coefficient

13 of the first group as a function of the number of iterations. This figure indicates that the

14 sensitivity coefficient with the source perturbation converges approximately after 7

15 iterations. The results with DANTSYS agree in most cases with those with the Monte

16 Carlo method within two standard deviations. Consequently, the Monte Carlo method

17 that incorporates the source perturbation method is verified through comparison with

18 the deterministic method.

\subsection{Superhistory method}

The superhistory method for incorporating the source perturbation effect into the differential operator method is applied for the numerical tests in Sec. 3.1. The sensitivity coefficient calculated by Eq. (20) approaches the converged value with the supergenerations. Fig. 3 shows the convergence situation of the sensitivity coefficients with the superhistory method for the perturbation of the cross sections in the first group. 
1 For the convergence of the sensitivity coefficients, 10 supergenerations seem to be 2 sufficient (Fig. 3).

3

[Table 6][Table 7][Table 8][Table 9]

The relative figures of merit for the perturbation in the first group (Table 10) for several supergenerations. The figure of merit is defined by

$$
\mathrm{FOM}=\frac{1}{s^{2} T},
$$

where $s=$ one standard deviation of the sensitivity coefficient and $T=$ computation time. Table 10 shows the FOM decreases with the supergeneration. The relative FOM of the superhistory method after 10 supergenerations is $0.4 \sim 0.8$ compared with the source perturbation iteration method. In the superhistory method, the information regarding the sensitivity coefficient is transferred between supergenerations, which makes the variance enlarge every time the supergeneration is updated. The superhistory method can reduce the memory requirement regardless of the decrease in the computational efficiency. Fig. 3 shows the sensitivity coefficient almost converges after the 8 th supergeneration. If the supergeneration is updated even after the desirable convergence is reached, it would only exacerbate the computational efficiency without improving the accuracy. Thus, as soon as the sensitivity coefficient reaches the convergence, the supergeneration should be terminated to minimize the reduction of the computational efficiency. 


\section{4. Conclusions}

3 The differential operator method is an easy and fast method to calculate the

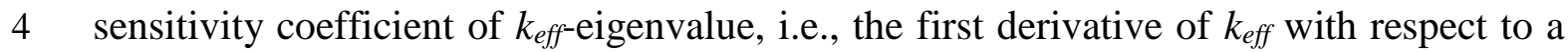

5 cross section if the effect of the source perturbation is negligibly small. However, if the

6 cross section whose sensitivity coefficient is sought to be calculated is localized, the

7 source perturbation effect significantly affects the sensitivity coefficient. To incorporate

8 the effect of the source perturbation in the sensitivity coefficient calculation, an iteration

9 procedure needs to be introduced like the adjoint-based method such as the iterated

10 fission probability (IFP) method that requires the fission chain to be followed over

11 generations. The differential operator method with the source perturbation iteration

12 requires a huge memory as with the IFP method if the sensitivity coefficients for a large

13 number of isotopes and energy groups are calculated at the same time. The superhistory

14 method, which has been previously adopted in other Monte Carlo techniques for the memory reduction technique, is introduced into the differential operator method. The sensitivity coefficient that includes the source perturbation effect can be calculated in a single particle history by tracking it over approximately ten supergenerations. There is no need to store a large amount of information until the end of a cycle. Thus, the memory requirement can be reduced per reaction by a factor of (particle histories per cycle $) \times($ the number of iterations for source perturbation, $\sim 10)$. The FOM decreases with the number of supergenerations. However, the reduction in the computational efficiency is not so large and may be acceptable. After the source perturbation effect converges, updating the supergeneration would increase the variance of the sensitivity coefficient. The iteration of the supergenerations should be terminated to optimize the computational efficiency after the convergence of the source perturbation is reached. 
1 Although this paper deals with the sensitivity coefficients with respect to the 2 multi-group macroscopic cross sections, the algorithm presented in this paper can be 3 applied straightforwardly to sensitivity analyses in the continuous energy Monte Carlo.

4 Future work will face the extension of the differential operator method to the 5 sensitivity coefficient calculation of general responses (i.e., the generalized sensitivity 6 coefficient). 


\section{$1 \quad$ References}

2 Alcouffe, R.E., Baker, R.S., Brinkley, F.W., Marr, D.R., O’Dell, R.D., Walters, W.F., 1995. DANTSYS: A diffusion accelerated neutral particle transport code system, LA-12969-M.

Aufiero, M., Bidaud, A., Hursin, M., Leppänen, J., Palmiotti, G., Pelloni, S., Rubiolo, P., 2015. A collision history-based approach to sensitivity/perturbation calculations in the continuous energy Monte Carlo code SERPENT, Ann. Nucl. Energy, 84, $245-258$.

Aufiero, M., Martin, M. Fratoni, M., 2016. XGPT: Extending Monte Carlo Generalized Perturbation Theory capabilities to continuous-energy sensitivity functions, Ann. Nucl. Energy, 96, 295-306.

Brissenden, R.J., Garlick, A.R., 1986. Biases in the estimation of $k_{\text {eff }}$ and its error by Monte Carlo methods. Ann. Nucl. Energy 113, 63-83.

Choi, S. H., Shim, H. J., 2016a. Memory-efficient calculations of adjoint-weighted tallies by the Monte Carlo Wielandt method, Ann. Nucl. Energy, 96, 287-294.

Choi, S. H., Shim, H. J., 2016b. Generalized sensitivity calculation in the Monte Carlo Wielandt method, Trans. Am. Nucl. Soc., 115, 575-577.

Densmore, J. D., McKinney, G.W., Hendricks, J.S., 1997. Correction to the MCNP perturbation feature for cross-section dependent tallies, LA-13374.

Jinaphanh, A., Leclaire, N., Cochet, B., 2016. Continuous-energy sensitivity coefficients in the MORET code, Nucl. Sci. Eng., 184, 53-68.

Kiedrowski, B.C., Brown, F.B., Wilson, P.P.H., 2011. Adjoint-weighted tallies for $k$-eigenvalue calculations with continuous-energy Monte Carlo. Nucl. Sci. Eng., 168 (3), 226-241.

Kiedrowski, B.C., Brown, F.B., 2013. Adjoint-based $k$-eigenvalue sensitivity 
1 coefficients to nuclear data using continuous-energy Monte Carlo. Nucl. Sci. Eng.

$2 \quad 174,227-244$.

3 McKinney, G.W., Iverson, J.L., 1996. Verification of the Monte Carlo differential operator technique for MCNP, Los Alamos National Laboratory, LA-13098.

Nagaya, Y., Mori, T., 2005. Impact of perturbed fission source on the effective multiplication factor in Monte Carlo perturbation calculations, J. Nucl. Sci. Technol., $42,428-441$.

Nagaya, Y., Mori, T., 2011. Estimation of sample reactivity worth with differential operator sampling method, Prog. Nucl. Sci. Technol., 2, 842-850.

Nagaya, Y., Okumura, K., Mori, T., 2015. Recent developments of JAEA's Monte Carlo code MVP for reactor physics applications, Ann., Nucl. Energy, 82, 85-89.

Perfetti, C. M., 2012. Advanced Monte Carlo methods for eigenvalue sensitivity coefficient calculations, Doctoral dissertation, University of Michigan.

Perfetti, C. M., Rearden, B. T., 2016. Development of a generalized perturbation theory method for sensitivity analysis using continuous-energy Monte Carlo methods, Nucl.

Qiu, Y., Liang, J., Wang, K., Yu, J., 2015. New strategies of sensitivity analysis capabilities in continuous-energy Monte Carlo code RMC. Ann. Nucl. Energy, 81, $50-61$.

Qiu, Y., Aufiero, M., Wang, K., Fratoni, M., 2016a. Development of sensitivity analysis capabilities of generalized responses to nuclear data in Monte Carlo code RMC, Ann. Nucl. Energy, 97, 142-152.

Qiu, Y., Shang, X., Tang, X., Liang, J., Wang, K., 2016b. Computing eigenvalue 
1

sensitivity coefficients to nuclear data by adjoint superhistory method and adjoint Wielandt method implemented in RMC code, Ann. Nucl. Eng., 87, 228-241.

Raskach, K. F., 2009. An improvement of the Monte Carlo generalized differential operator method by taking into account first- and second-order perturbations of fission source, Nucl. Sci. Eng., 162, 158-166.

Raskach, K. F., 2010. Extension of differential operator method to inhomogeneous problems with internal and external neutron sources. Nucl. Sci. Eng., 165, 320-330.

Rearden, B.T., 2004. Perturbation theory eigenvalue sensitivity analysis with MonteCarlo techniques. Nucl. Sci. Eng. 146, 367-382.

Rief, H., 1984. Generalized Monte Carlo perturbation algorithms for correlated sampling and a second-order Taylor series approach, Ann. Nucl. Energy, 9 $455-476$.

She, D., Wang, K., Yu, G., 2012. Asymptotic Wielandt method and superhistory method for source convergence in Monte Carlo criticality calculation, Nucl. Sci. Eng., $172,127-137$

Shim, H. J., Kim, C. H., 2011. Adjoint sensitivity and uncertainty analyses in Monte Carlo forward calculations, J. Nucl. Sci. Technol., 48 (12), 1453-1461.

Terranova, N., Zoia, A., 2017. Generalized iterated fission probability for Monte Carlo eigenvalue calculations, Ann. Nucl. Energy, 108, 57-66.

Truchet, G., Leconte, P., Santamarina, A., Damian, F., Zoia, A., 2015. Computing adjoint-weighted kinetics parameters in TRIPOLI- $4^{\circledR}$ by the Iterated Fission Probability method, Ann. Nucl. Energy, 81, 17-26.

Wang, K., Li, Z., She, D., et al., 2015. RMC - a Monte Carlo code for reactor core analysis. Ann. Nucl. Energy 82, 121-129.

Williams, M. L., 1977. The relations between various contributon variables used in 
1 spatial channel theory applied to reactor shielding analysis, Nucl. Sci. Eng., 63, $2220-222$.

3 Yamamoto, T., Miyoshi, Y., 2004. Reliable method for fission source convergence of

4 Monte Carlo criticality calculation with Wielandt's method, J. Nucl. Sci. Technol., $5 \quad 41(2), 99-107$.

6

7 
2 Fig. 1 Geometry for the numerical tests.

3 Fig. 2 Convergence of the sensitivity coefficient with the source perturbation to the

4 scattering cross section in the first group. The standard deviation is smaller than the dot 5 size.

6 Fig. 3 Relative sensitivity coefficients to the cross sections in the first group vs.

7 supergeneration. $S_{\mathrm{sh}}$ : the superhistory method: $S_{\mathrm{nps}}$ without the source perturbation: $\mathrm{S}_{\mathrm{ps}}$ 8 with the source perturbation. 


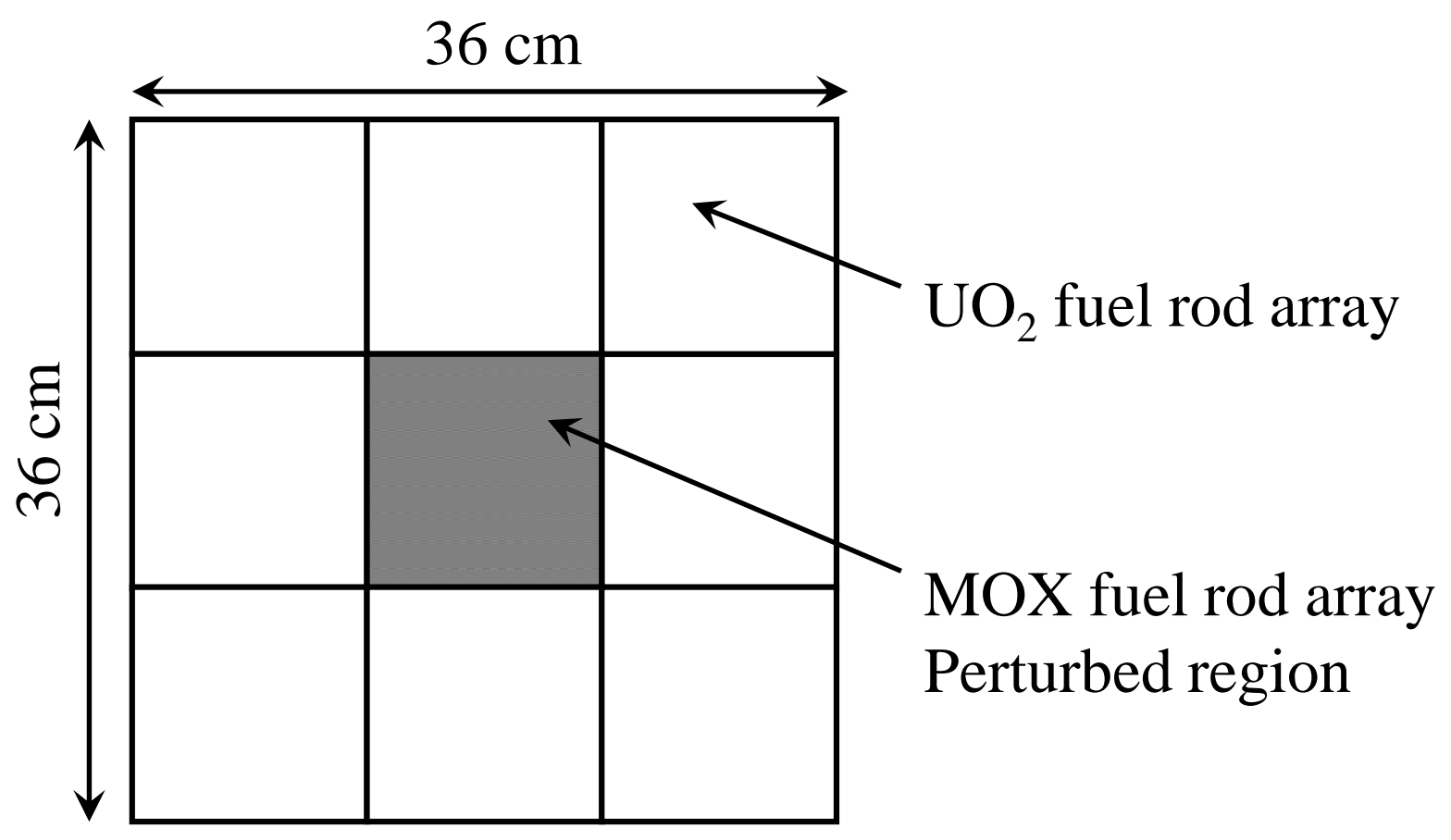

Fig. 1 Geometry for the numerical tests. 


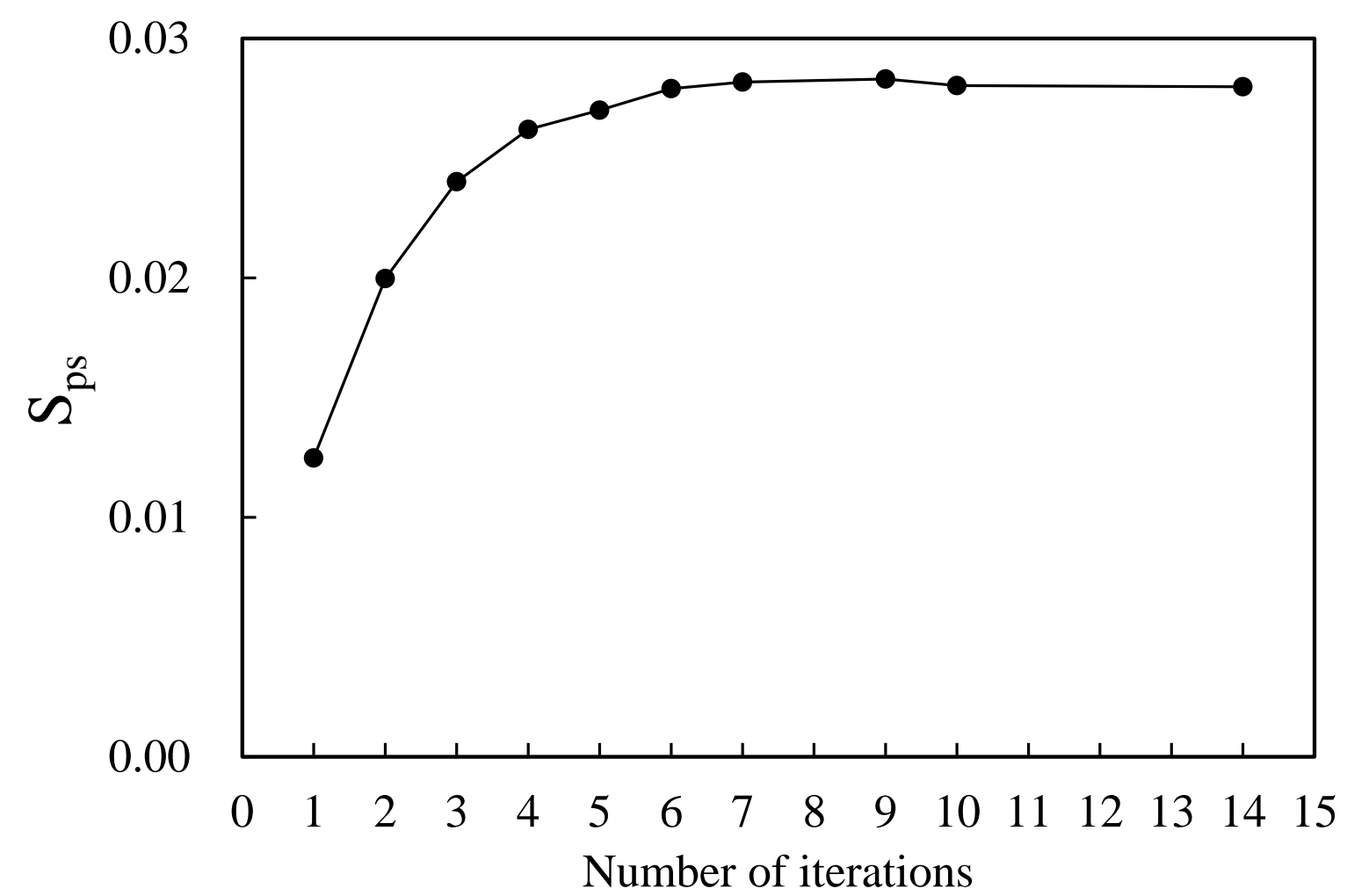

Fig. 2 Convergence of the sensitivity coefficient with the source perturbation to the scattering cross section in the first group. The standard deviation is smaller than the dot size. 


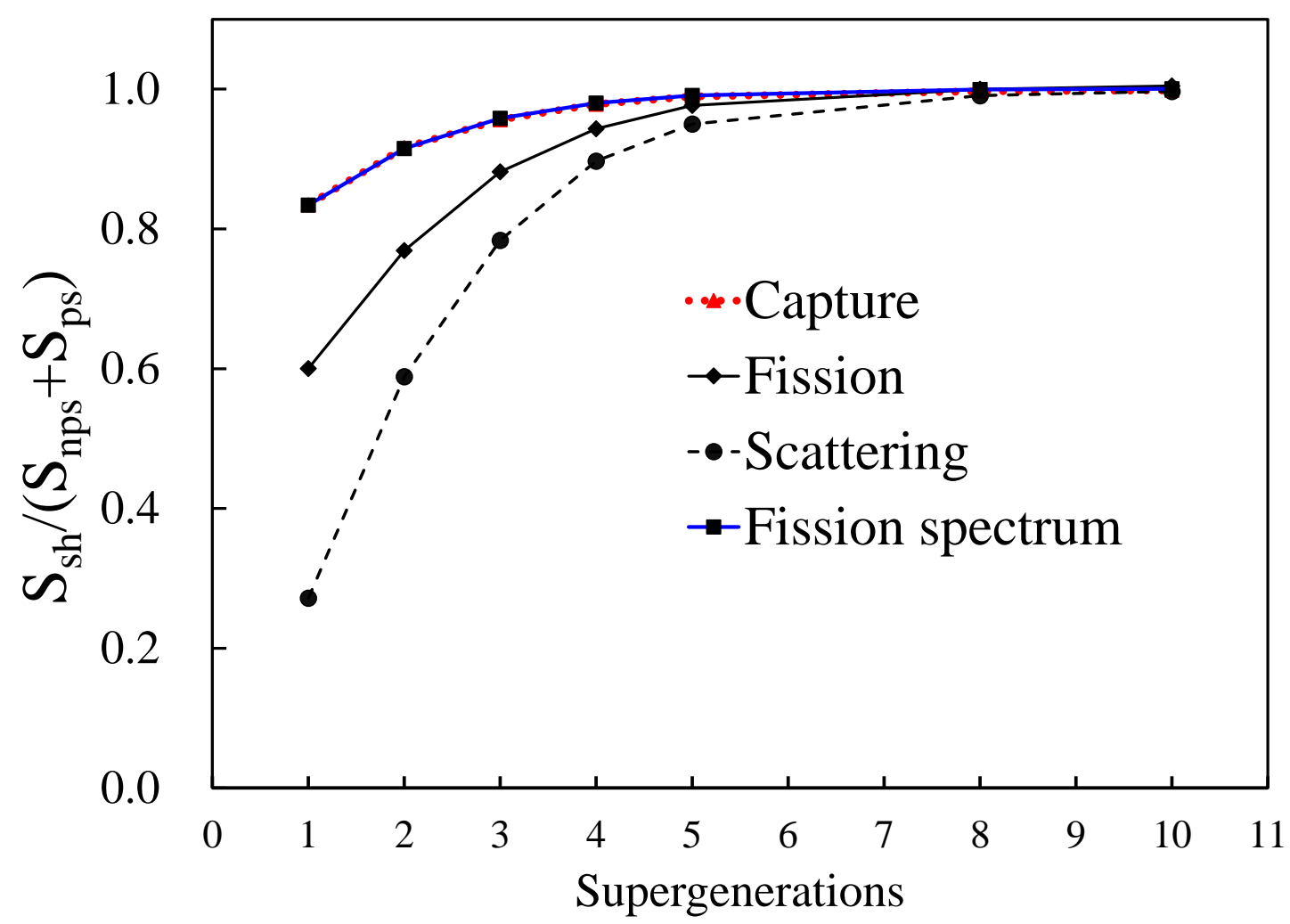

Fig. 3 Relative sensitivity coefficients to the cross sections in the first group vs. supergeneration. $S_{\mathrm{sh}}$ : the superhistory method: $S_{\mathrm{nps}}$ without the source perturbation: $S_{\mathrm{ps}}$ with the source perturbation. 
Table 1 Three-group constants for $\mathrm{UO}_{2}$ fuel rod array and MOX fuel rod array

\begin{tabular}{|c|c|c|c|}
\hline & & $\begin{array}{c}\mathrm{UO}_{2} \text { fuel rod } \\
\text { array }\end{array}$ & $\begin{array}{c}\text { MOX fuel rod } \\
\text { array }\end{array}$ \\
\hline \multirow{3}{*}{$\begin{array}{l}\text { Total cross } \\
\text { section }\end{array}$} & $\Sigma_{t 1}\left(\mathrm{~cm}^{-1}\right)$ & 0.29829 & 0.289397 \\
\hline & $\Sigma_{t 2}\left(\mathrm{~cm}^{-1}\right)$ & 0.83334 & 0.825987 \\
\hline & $\Sigma_{t 3}\left(\mathrm{~cm}^{-1}\right)$ & 1.6389 & 1.6600 \\
\hline \multirow{3}{*}{$\begin{array}{l}\text { Fission cross } \\
\text { section }\end{array}$} & $\Sigma_{f 1}\left(\mathrm{~cm}^{-1}\right)$ & 0.0030586 & 0.0025989 \\
\hline & $\Sigma_{f 2}\left(\mathrm{~cm}^{-1}\right)$ & 0.0021579 & 0.0019544 \\
\hline & $\Sigma_{f 3}\left(\mathrm{~cm}^{-1}\right)$ & 0.056928 & 0.070119 \\
\hline \multirow{3}{*}{$\begin{array}{l}\text { Absorption } \\
\text { cross section }\end{array}$} & $\Sigma_{a 1}\left(\mathrm{~cm}^{-1}\right)$ & 0.003385 & 0.003265 \\
\hline & $\Sigma_{a 2}\left(\mathrm{~cm}^{-1}\right)$ & 0.011895 & 0.011435 \\
\hline & $\Sigma_{a 3}\left(\mathrm{~cm}^{-1}\right)$ & 0.086180 & 0.12441 \\
\hline \multirow{2}{*}{$\begin{array}{l}\text { Group transfer } \\
\text { cross section }\end{array}$} & $\Sigma_{s}^{1 \rightarrow 2}\left(\mathrm{~cm}^{-1}\right)$ & 0.073843 & 0.071620 \\
\hline & $\Sigma_{s}^{2 \rightarrow 3}\left(\mathrm{~cm}^{-1}\right)$ & 0.043803 & 0.044045 \\
\hline $\begin{array}{l}\text { Neutrons per } \\
\text { fission }\end{array}$ & $v$ & 2.4 & 2.8 \\
\hline \multirow{3}{*}{$\begin{array}{l}\text { Fission } \\
\text { spectrum }\end{array}$} & $\chi_{1}$ & 0.878198 & 0.878198 \\
\hline & $\chi_{2}$ & 0.121802 & 0.121802 \\
\hline & $\chi_{3}$ & 0 & 0 \\
\hline
\end{tabular}


Table 2 Sensitivity coefficients to the capture cross section.

\begin{tabular}{lccc}
\hline & 1 st Gr. & 2nd Gr. & 3rd Gr. \\
\hline DANTSYS & $-1.0603 \times 10^{-3}$ & $-5.0217 \times 10^{-2}$ & $-1.3207 \times 10^{-1}$ \\
\hline Differential operator (MC) & & & \\
Without source perturbation & $-8.8576 \times 10^{-4}$ & $-3.8184 \times 10^{-2}$ & $-9.3008 \times 10^{-2}$ \\
& $\left(2.2 \times 10^{-7}\right)^{*}$ & $\left(5 \times 10^{-6}\right)$ & $\left(2.3 \times 10^{-5}\right)$ \\
Source perturbation effect & $-1.7581 \times 10^{-4}$ & $-1.2071 \times 10^{-2}$ & $-3.9113 \times 10^{-2}$ \\
& $\left(4.5 \times 10^{-7}\right)$ & $\left(1.4 \times 10^{-5}\right)$ & $\left(6.5 \times 10^{-5}\right)$ \\
Total & $-1.0616 \times 10^{-3}$ & $-5.0255 \times 10^{-2}$ & $-1.3212 \times 10^{-1}$ \\
& $\left(5.0 \times 10^{-7}\right)$ & $\left(1.5 \times 10^{-5}\right)$ & $\left(7 \times 10^{-5}\right)$ \\
\hline MC/DANTSYS & 1.001 & 1.001 & 1.000 \\
& $(0.001)$ & $(0.001)$ & $(0.001)$ \\
\hline
\end{tabular}

*one standard deviation 
Table 3 Sensitivity coefficients to the fission cross section.

\begin{tabular}{lccc}
\hline & 1 st Gr. & 2nd Gr. & 3rd Gr. \\
\hline DANTSYS & $1.4185 \times 10^{-2}$ & $1.3389 \times 10^{-2}$ & $1.3880 \times 10^{-1}$ \\
\hline Differential operator (MC) & & & \\
Without source perturbation & $8.4897 \times 10^{-3}$ & $8.4553 \times 10^{-3}$ & $9.2866 \times 10^{-2}$ \\
& $\left(1.3 \times 10^{-6}\right)^{*}$ & $\left(2.1 \times 10^{-6}\right)$ & $\left(2.2 \times 10^{-5}\right)$ \\
Source perturbation effect & $5.6517 \times 10^{-3}$ & $4.9375 \times 10^{-3}$ & $4.6046 \times 10^{-2}$ \\
& $\left(1.64 \times 10^{-5}\right)$ & $\left(3.01 \times 10^{-5}\right)$ & $\left(9.0 \times 10^{-5}\right)$ \\
Total & $1.4141 \times 10^{-2}$ & $1.3393 \times 10^{-2}$ & $1.3891 \times 10^{-1}$ \\
& $\left(1.6 \times 10^{-5}\right)$ & $\left(3.0 \times 10^{-5}\right)$ & $\left(9 \times 10^{-5}\right)$ \\
\hline MC/DANTSYS & 0.997 & 1.000 & 1.001 \\
& $(0.001)$ & $(0.002)$ & $(0.001)$ \\
\hline
\end{tabular}

*one standard deviation 
Table 4 Sensitivity coefficients to the scattering cross section.

\begin{tabular}{lccc}
\hline & 1 st Gr. & 2nd Gr. & 3rd Gr. \\
\hline DANTSYS & $3.8192 \times 10^{-2}$ & $6.6733 \times 10^{-2}$ & $* *$ \\
\hline Differential operator (MC) & & & \\
Without source perturbation & $1.0443 \times 10^{-2}$ & $3.2650 \times 10^{-2}$ & $1.5980 \times 10^{-2}$ \\
& $\left(9.0 \times 10^{-5}\right)^{*}$ & $\left(9.3 \times 10^{-5}\right)$ & $\left(8.0 \times 10^{-5}\right)$ \\
Source perturbation effect & $2.7983 \times 10^{-2}$ & $3.4129 \times 10^{-2}$ & $6.9639 \times 10^{-3}$ \\
& $\left(1.93 \times 10^{-4}\right)$ & $\left(2.26 \times 10^{-4}\right)$ & $\left(2.390 \times 10^{-4}\right)$ \\
Total & $3.8427 \times 10^{-2}$ & $6.6780 \times 10^{-2}$ & $2.2944 \times 10^{-2}$ \\
& $\left(2.13 \times 10^{-4}\right)$ & $\left(2.44 \times 10^{-4}\right)$ & $\left(2.52 \times 10^{-4}\right)$ \\
\hline MC/DANTSYS & 1.006 & 1.001 & - \\
\hline
\end{tabular}

*one standard deviation

** This result is incorrect and omitted because of the small value in the numerator of Eq. (21). 
Table 5 Sensitivity coefficients to the fission spectrum.

\begin{tabular}{lcc}
\hline & 1 st Gr. & 2nd Gr. \\
\hline DANTSYS & $3.0664 \times 10^{-1}$ & $4.8818 \times 10^{-2}$ \\
\hline Differential operator (MC) & & \\
Without source perturbation & $2.5581 \times 10^{-1}$ & $3.7192 \times 10^{-2}$ \\
& $\left(7 \times 10^{-5}\right)^{*}$ & $\left(2.1 \times 10^{-5}\right)$ \\
Source perturbation effect & $5.0886 \times 10^{-2}$ & $1.1721 \times 10^{-2}$ \\
& $\left(1.26 \times 10^{-4}\right)$ & $\left(4.6 \times 10^{-5}\right)$ \\
Total & $3.0669 \times 10^{-1}$ & $4.8913 \times 10^{-2}$ \\
& $\left(1.42 \times 10^{-4}\right)$ & $\left(5.1 \times 10^{-5}\right)$ \\
\hline MC/DANTSYS & 1.000 & 1.001 \\
& $(0.001)$ & $(0.001)$ \\
\hline
\end{tabular}

*one standard deviation 
Table 6 Sensitivity coefficients to the capture cross section with the superhistory method.

\begin{tabular}{|c|c|c|c|c|}
\hline & & 1st Gr. & 2nd Gr. & 3rd Gr. \\
\hline DANTSYS & & $-1.0603 \times 10^{-3}$ & $-5.0217 \times 10^{-2}$ & $-1.3207 \times 10^{-1}$ \\
\hline \multicolumn{5}{|l|}{ Differential operator (MC) } \\
\hline $\begin{array}{l}\text { Source perturbation } \\
\text { method }\end{array}$ & iteration & $\begin{array}{c}-1.0616 \times 10^{-3} \\
\left(5.0 \times 10^{-7}\right)^{*}\end{array}$ & $\begin{array}{r}-5.0255 \times 10^{-2} \\
\left(1.5 \times 10^{-5}\right)\end{array}$ & $\begin{array}{c}-1.3212 \times 10^{-1} \\
\left(7 \times 10^{-5}\right)\end{array}$ \\
\hline Superhistory method & & $-1.0602 \times 10^{-3}$ & $-5.0247 \times 10^{-2}$ & $-1.3229 \times 10^{-1}$ \\
\hline 10 supergenerations & & $\left(6.9 \times 10^{-7}\right)$ & $\left(3.3 \times 10^{-5}\right)$ & $\left(1.0 \times 10^{-4}\right)$ \\
\hline \multirow{2}{*}{\multicolumn{2}{|c|}{ MC/DANTSYS }} & 1.000 & 1.001 & 1.002 \\
\hline & & $(0.001)$ & $(0.001)$ & $(0.001)$ \\
\hline
\end{tabular}

*one standard deviation 
Table 7 Sensitivity coefficients to the fission cross section with the superhistory method.

\begin{tabular}{|c|c|c|c|c|}
\hline & & 1st Gr. & 2nd Gr. & 3rd Gr. \\
\hline DANTSYS & & $1.4185 \times 10^{-2}$ & $1.3389 \times 10^{-2}$ & $1.3880 \times 10^{-1}$ \\
\hline \multicolumn{5}{|l|}{ Differential operator (MC) } \\
\hline $\begin{array}{l}\text { Source perturbation } \\
\text { method }\end{array}$ & iteration & $\begin{array}{l}1.4141 \times 10^{-2} \\
\left(1.6 \times 10^{-5}\right)^{*}\end{array}$ & $\begin{array}{l}1.3393 \times 10^{-2} \\
\left(3.0 \times 10^{-5}\right)\end{array}$ & $\begin{array}{c}1.3891 \times 10^{-1} \\
\left(9 \times 10^{-5}\right)\end{array}$ \\
\hline Superhistory method & & $1.4195 \times 10^{-2}$ & $1.3320 \times 10^{-2}$ & $1.3880 \times 10^{-1}$ \\
\hline 10 supergenerations & & $\left(5.0 \times 10^{-5}\right)$ & $\left(4.9 \times 10^{-5}\right)$ & $\left(1.4 \times 10^{-4}\right)$ \\
\hline \multirow{2}{*}{\multicolumn{2}{|c|}{ MC/DANTSYS }} & 1.001 & 0.995 & 1.000 \\
\hline & & $(0.003)$ & $(0.004)$ & $(0.001)$ \\
\hline
\end{tabular}

*one standard deviation 
Table 8 Sensitivity coefficients to the scattering cross section with the superhistory method.

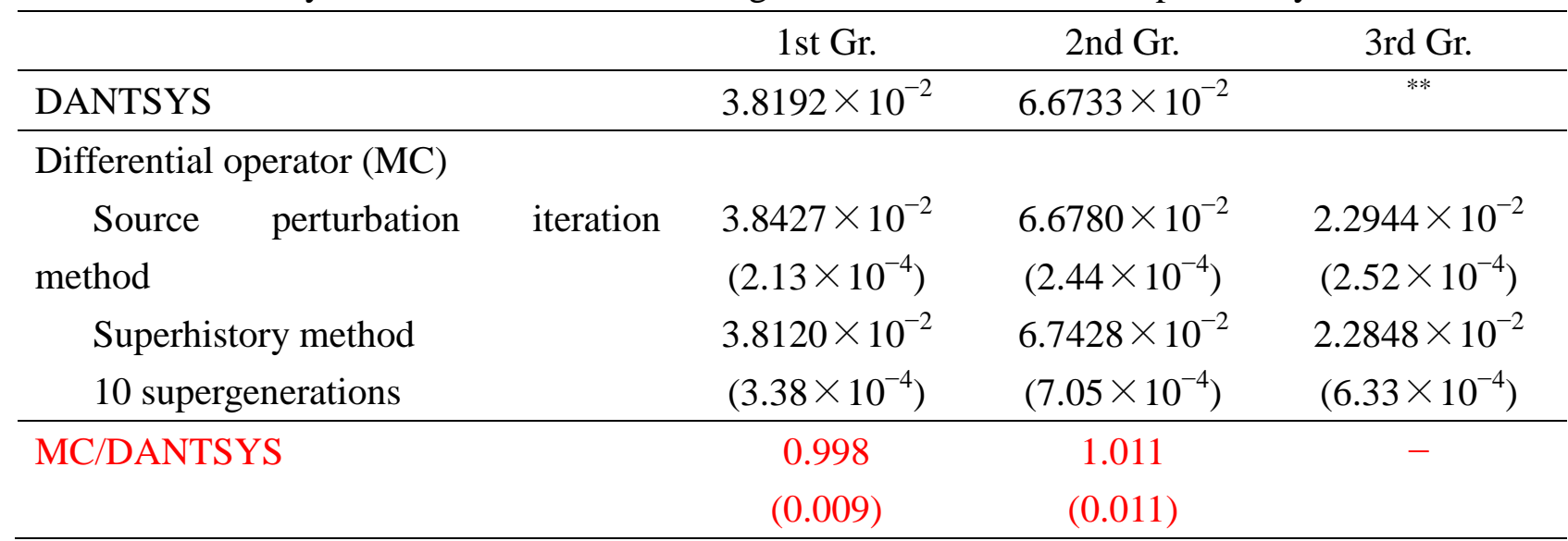

*one standard deviation

** This result is incorrect and omitted because of the small value in the numerator of Eq. (21). 
Table 9 Sensitivity coefficients to the fission spectrum with the superhistory method.

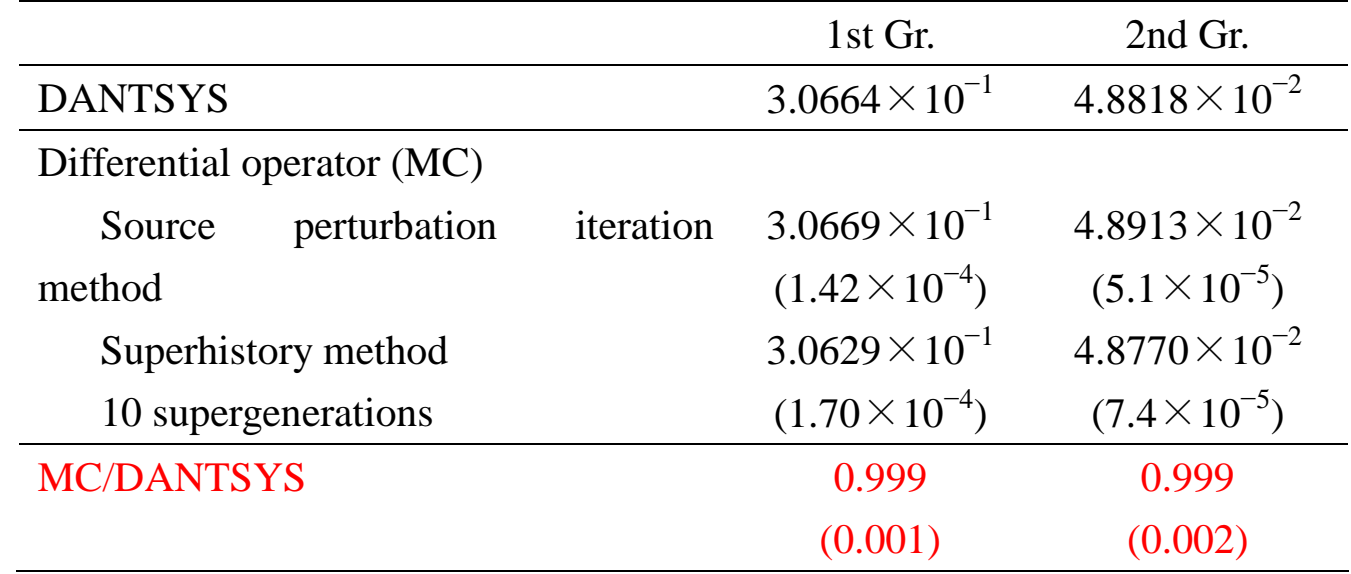

*one standard deviation 
Table 10 Relative figure of merit of the superhistory method for the first group perturbations.

\begin{tabular}{lcccc}
\hline & Capture & Fission & Scattering & $\begin{array}{c}\text { Fission } \\
\text { spectrum }\end{array}$ \\
\hline Source perturbation iteration method & 1.000 & 1.000 & 1.000 & 1.000 \\
2 supergenerations & 3.102 & 4.842 & 2.540 & 3.275 \\
3 supergenerations & 2.017 & 2.208 & 1.656 & 2.387 \\
4 supergenerations & 1.398 & 1.298 & 1.283 & 1.814 \\
5 supergenerations & 1.193 & 0.883 & 0.949 & 1.424 \\
8 supergenerations & 0.822 & 0.526 & 0.553 & 1.030 \\
10 supergenerations & 0.607 & 0.397 & 0.453 & 0.789 \\
\hline
\end{tabular}

